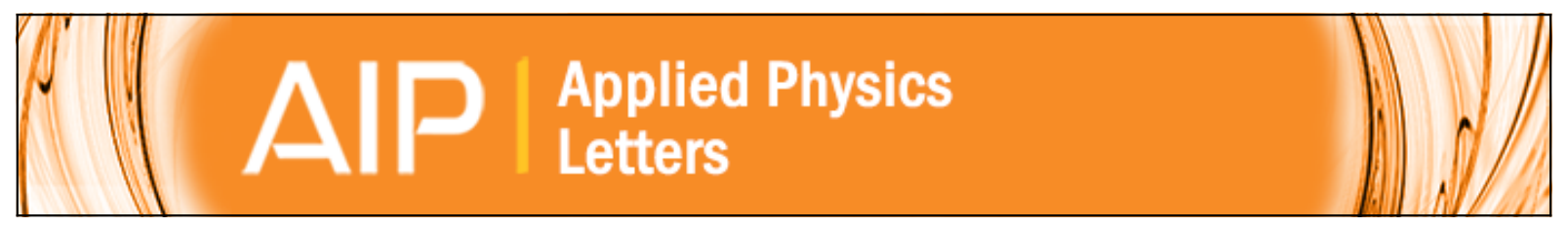

\title{
Polarized photoluminescence and time-resolved photoluminescence from single CdS
} nanosheets

T. B. Hoang, L. V. Titova, A. Mishra, L. M. Smith, H. E. Jackson, K.-Y. Lee, H. Rho, J. M. Yarrison-Rice, Y.-J. Choi, K. J. Choi, and J.-G. Park

Citation: Applied Physics Letters 92, 143112 (2008); doi: 10.1063/1.2907507

View online: http://dx.doi.org/10.1063/1.2907507

View Table of Contents: http://scitation.aip.org/content/aip/journal/apl/92/14?ver=pdfcov

Published by the AIP Publishing

\section{Articles you may be interested in}

Energy transfer from semiconductor nanocrystal monolayers to metal surfaces revealed by time-resolved photoluminescence spectroscopy

Appl. Phys. Lett. 92, 133118 (2008); 10.1063/1.2906369

Spatially resolved photoluminescence mapping of single CdS nanosheets

Appl. Phys. Lett. 92, 013111 (2008); 10.1063/1.2828707

Low-temperature photoluminescence imaging and time-resolved spectroscopy of single CdS nanowires

Appl. Phys. Lett. 89, 053119 (2006); 10.1063/1.2266414

Excitons and surface luminescence of CdS nanoribbons

Appl. Phys. Lett. 84, 795 (2004); 10.1063/1.1644625

Photoluminescence spectrum of highly excited single CdS nanocrystals studied by a scanning near-field optical microscope

Appl. Phys. Lett. 81, 141 (2002); 10.1063/1.1490141

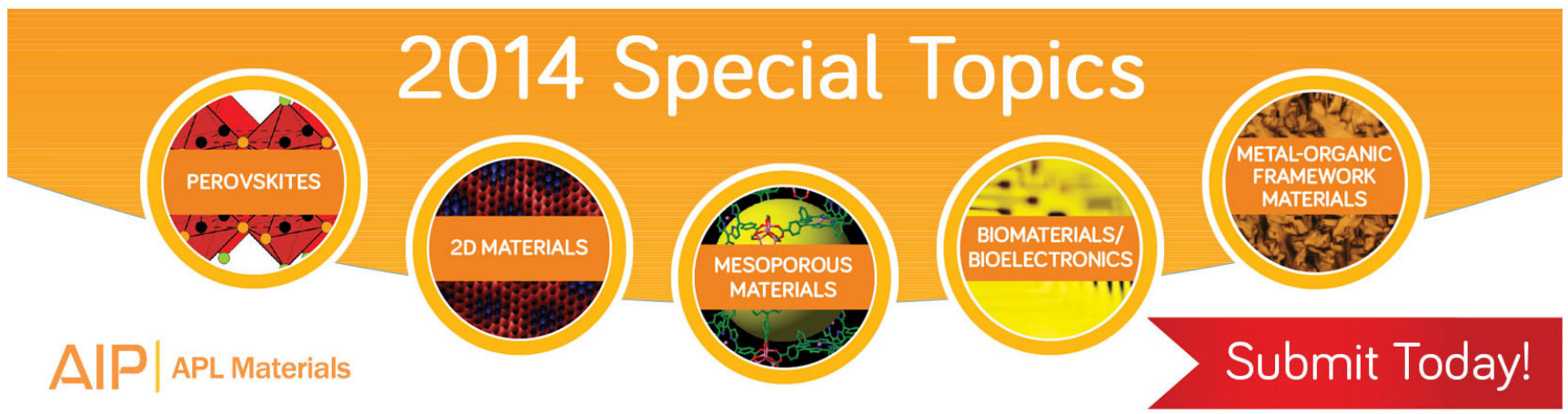




\title{
Polarized photoluminescence and time-resolved photoluminescence from single CdS nanosheets
}

\author{
T. B. Hoang, ${ }^{1}$ L. V. Titova, ${ }^{1}$ A. Mishra, ${ }^{1}$ L. M. Smith, ${ }^{1}$ H. E. Jackson, ${ }^{1, a)}$ K.-Y. Lee, ${ }^{2}$ \\ H. Rho, ${ }^{2, a)}$ J. M. Yarrison-Rice, ${ }^{3}$ Y.-J. Choi, ${ }^{4}$ K. J. Choi, ${ }^{4}$ and J.-G. Park ${ }^{4}$ \\ ${ }^{1}$ Department of Physics, University of Cincinnati, Cincinnati, Ohio 45221, USA \\ ${ }^{2}$ Department of Physics, Chonbuk National University, Jeonju 561-756, Republic of Korea \\ ${ }^{3}$ Physics Department, Miami University, Oxford, Ohio 45056, USA \\ ${ }^{4}$ Nano Materials Research Center, Korea Institute of Science and Technology, Seoul 130-650, Republic of \\ Korea
}

(Received 10 January 2008; accepted 6 March 2008; published online 10 April 2008)

\begin{abstract}
We have utilized polarized low temperature photoluminescence (PL) to probe the electronic states and structural symmetries of individual $\mathrm{CdS}$ nanosheets. High resolution transmission electron microscopy measurements indicate highly crystalline material with different nanosheets exhibiting significant variations of the direction of the $c$ axis, which are consistent with polarization measurements of PL from single CdS nanosheets. The quality of the nanosheets is reflected in measurements of exciton lifetimes of $\sim 200 \mathrm{ps}$, a value significantly longer than observed for CdS nanowires whose diameter is the same as the thickness of these nanosheets, but shorter than that observed in bulk crystals. () 2008 American Institute of Physics. [DOI: 10.1063/1.2907507]
\end{abstract}

The spatial confinement of carriers in many nanoscale semiconductor structures makes them highly attractive because of their unusual optical and electrical properties. Semiconductor nanosheets (NSs) are particularly appealing because while they have one dimension in the nanoscale range, their other two dimensions are in the micrometer range making them easier to incorporate into existing or new optoelectronic device structures. ${ }^{1-9}$ NSs now form the basis for a number of optoelectronic devices including field effect transistors and light emitting diodes. ${ }^{1,5,6,9}$ The rapid development of applications for NSs, including CdS NSs, indicates that even more striking uses for these novel nanostructures might be possible if growth conditions were optimized and their electronic states and structural symmetries understood.

In this letter, we demonstrate that low temperature polarized micro-photoluminescence (micro-PL) is a powerful method that can be used to determine the $c$ axis of $\mathrm{CdS}$ single NSs. In addition, time-resolved PL measurements provide a characterization of both the quality and the low surface recombination velocity of these single NSs, measures that are important for potential applications. As we will show, high resolution transmission electron microscope (HRTEM) images provide evidence that the NSs are single crystalline with hexagonal wurtzite structure. Time-resolved PL measurements give exciton recombination lifetimes of $\sim 200$ ps which are significantly longer than that of the near band edge emission recently observed in single $\mathrm{CdS}$ nanowires $(<50 \mathrm{ps}),{ }^{10,11}$ but shorter than the lifetimes observed in bulk $\mathrm{CdS}$ crystals.

The catalyst-assisted vapor phase transport growth method was used to grow the CdS NSs. ${ }^{12}$ The size and shape of the NSs are quite uniform with individual NSs $\sim 5 \mu \mathrm{m}$ in width, $\sim 40-50 \mathrm{~nm}$ in thickness, and up to several tens of micrometers in length, all dimensions much larger than the

\footnotetext{
a) Authors to whom correspondence should be addressed. Electronic
} addresses: howard.jackson@uc.edu and rho@chonbuk.ac.kr.
CdS Bohr radius $(3 \mathrm{~nm})$ and thus no quantum confinement effects are expected.

To measure single structures, the NSs were dispersed from the growth substrate into an ethanol solution and then deposited onto either a silicon substrate or a TEM microgrid, resulting in a dilute array of NSs with an average separation of hundreds of micrometers. The crystallinity and structure of individual CdS NSs were investigated by HR-TEM. Figure 1(a) shows the end of a CdS NS, with an enlarged image of the region noted by the square box. The prominent wrinkling of the NS surface in the large-field image may be indicative of strain resulting from the Van der Waal's forces which hold the NS flat to the substrate. The HR-TEM image in the lower inset of Fig. 1(a) shows a highly crystalline wurtzite structure with the $c$ axis approximately perpendicular to the long axis of the CdS NS. Figure 1(b) shows a different CdS NS of similarly highly crystalline wurtzite structure, however, with the $c$ axis not perpendicular to the long axis of the NS.

Low temperature PL measurements were taken either with a cw argon ion laser or with a $200 \mathrm{fs,} 432 \mathrm{~nm}$ frequency-doubled Ti-sapphire mode-locked laser. The NSs dispersed on a Si substrate are placed onto the cold finger of a continuous-flow helium cryostat. A $50 \times / 0.5 \mathrm{NA}$ (numerical aperture) long working length microscope objective was utilized for both illumination and collection of the PL from a single NS. The polarization of the excitation could be controlled and the emitted PL analyzed using a matched set of Glan-Thomson linear polarizers and Babinet-Soleil compensators. The PL was dispersed by a triple spectrometer working in subtractive mode, and detected either by a 2000 $\times 800$ pixel liquid nitrogen-cooled charge-coupled device detector for $\mathrm{cw}$ measurements or a fast microchannel plate phototube for pulsed measurements. Time resolution was obtained using time-correlated single photon counting techniques. ${ }^{13}$ The temporal system response was measured to be $<80 \mathrm{ps}$, and the optical resolution and laser focal spots were $1.5 \mu \mathrm{m}$. All measurements were conducted at $15 \mathrm{~K}$. 

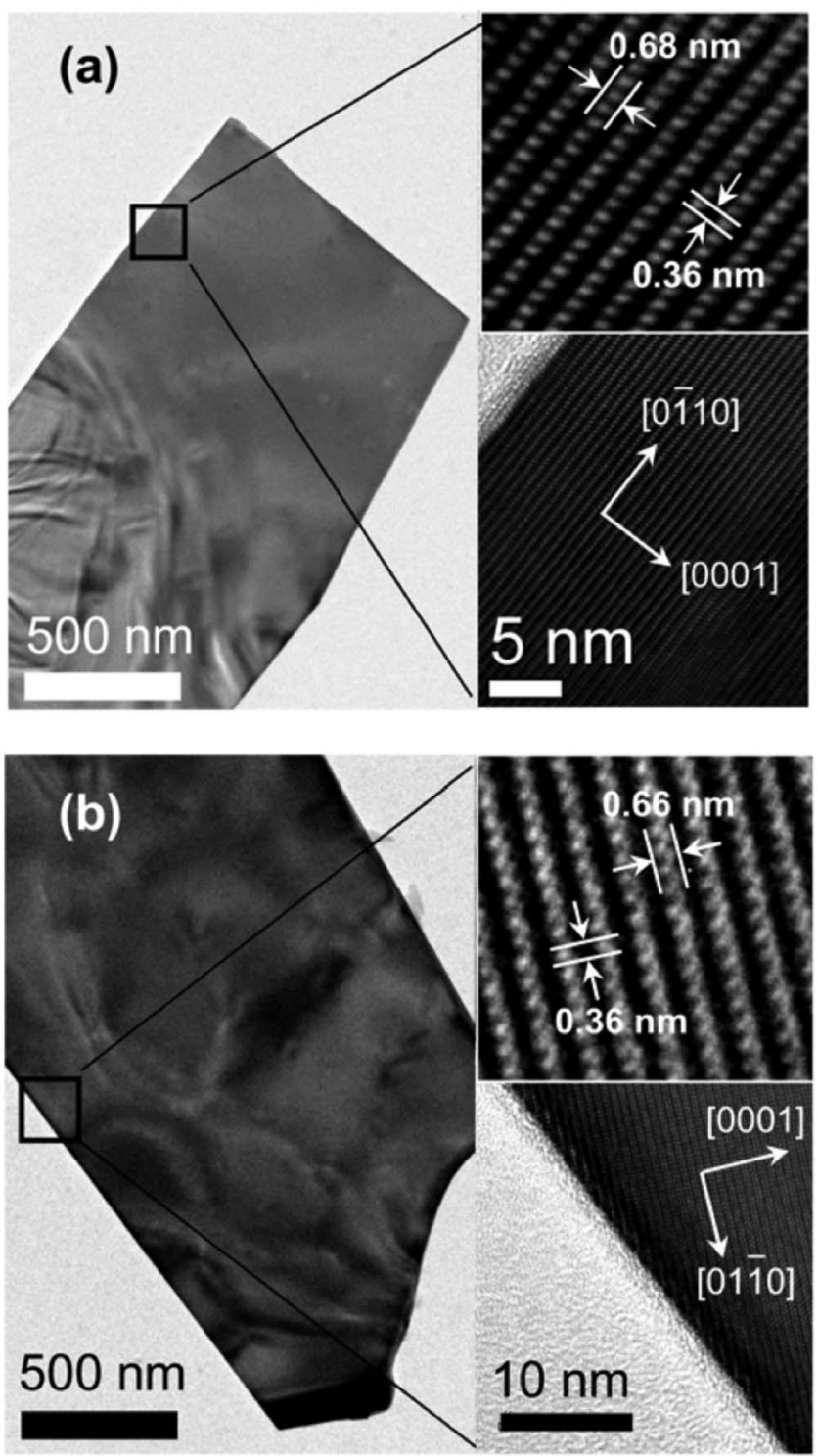

FIG. 1. Low and high magnification TEM images of two different CdS NSs. Insets to figures on right side show the HR-TEM image of the NS with fast Fourier transformation images. Note that the crystallographic $c$ axis is not always perpendicular to the long axis of the NS.

In Figs. 2(a) and 2(b), we show typical low temperature cw PL spectra of two different single CdS NSs. Spectra were measured with the excitation laser circularly polarized, while the emission passed through a linear polarization analyzer at different angles measured from $0^{\circ}\left(0^{\circ}\right.$ is alignment parallel to the long axis of the NS). The spectra are characterized by a relatively narrow peak at $2.547 \mathrm{eV}$ [full width at half maximum $(\mathrm{FWHM}) \sim 4 \mathrm{meV}]$ and a much broader emission near $2.563 \mathrm{eV}$ (FHWM $\sim 11 \mathrm{meV}$ ). This broader emission peak varies in energy in different NSs and often displays internal structure due to contributions from different exciton states. The known exciton lines for $\mathrm{CdS}$ include an $A$ free exciton at $2.553 \mathrm{eV}$ and a $B$ free exciton at $2.569 \mathrm{eV}$. Two bound exciton states have been recorded in CdS. The narrow peak at $2.547 \mathrm{eV}$ is consistent with the $I_{2 A}$ recombination line from an $A$ exciton bound to a neutral donor. The $I_{2 B}$ line would result from the $B$ exciton-neutral donor recombination. ${ }^{14,15}$ The energies for these four known states are marked by vertical lines on each of the spectra in Figs. 2(a) and 2(b).

Both free and bound excitons associated with the $A$ - and $B$-hole bands of wurtzite $\mathrm{CdS}$ recombine and emit strongly
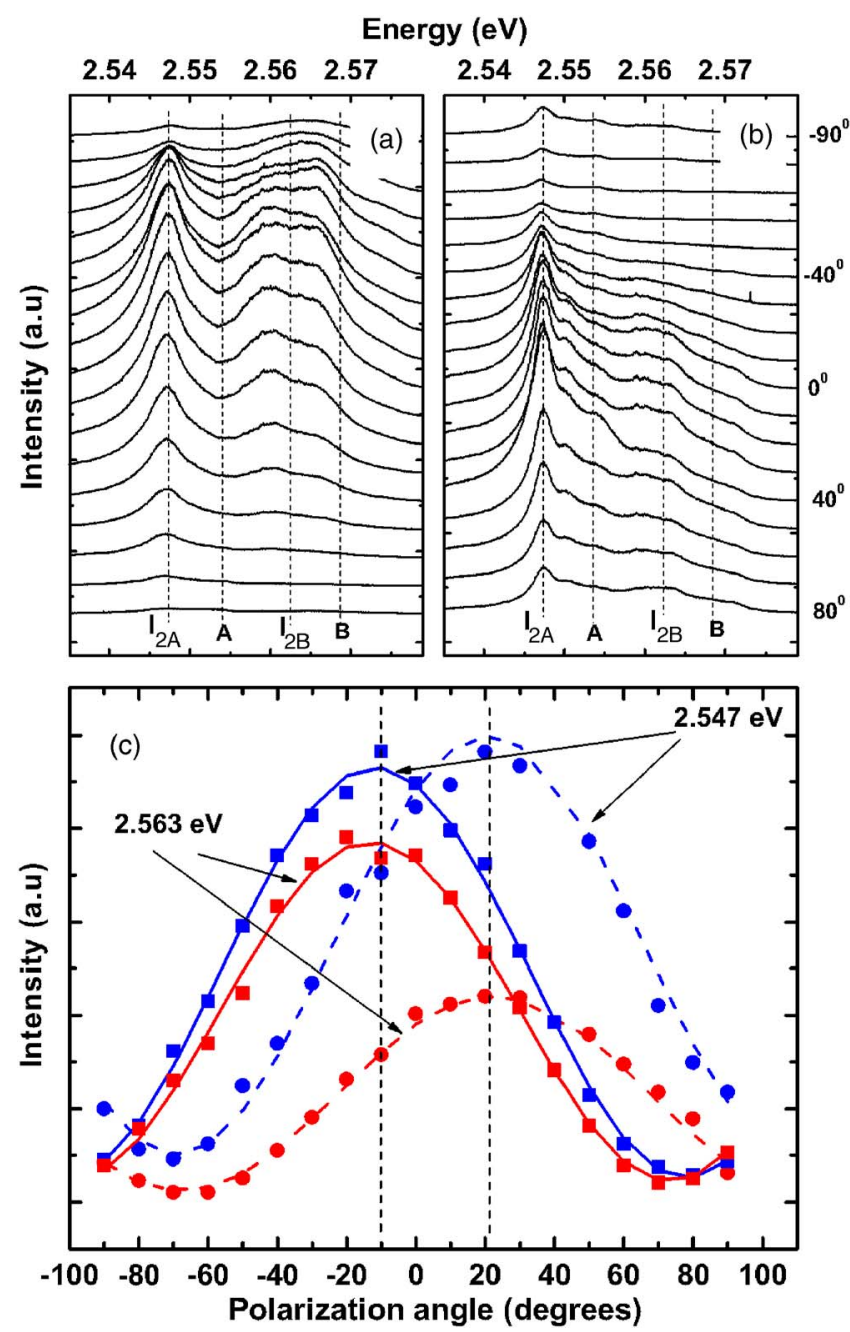

FIG. 2. (Color online) [(a) and (b)] PL spectra of two different CdS NSs. (c) PL intensity as a function of detection angle of the PL emission at 2.547 and $2.563 \mathrm{eV}$ from these two NSs (squares and circles, respectively).

polarized perpendicular to the $c$ axis. ${ }^{14,15}$ Figure 2(c) displays the peak PL emission intensities of the two respective NSs (squares and circles, respectively) at 2.547 and $2.563 \mathrm{eV}$ as a function of the angle of the linear polarization analyzer relative to the long axis of the NS. The red and blue curves are fits to $\cos ^{2} \theta$. We see that the emission of both peaks is strongly polarized. In the first NS [Figs. 2(a) and 2(c)], the maximum intensity occurs in the direction nearly parallel $\left(-10^{\circ}\right)$ to the NS long axis. For a wurtzite structure, this indicates that the $c$ axis of this particular NS is almost perpendicular to the long axis of the NS. The second NS [Figs. 2(b) and 2(c)], displays a very different polarization response with a peak intensity at about $+20^{\circ}$ suggesting the $c$ axis in this second NS is $110^{\circ}$ from the NS long axis. This result is consistent with the variability shown by the TEM results. This also directly demonstrates that polarized PL measurements provide a convenient nondestructive way in which to determine a NS's crystal orientation.

To gain more insight into the quality of these NSs, we display time-resolved PL at one position centered on a single NS. The NS is excited by $200 \mathrm{fs}$ laser pulses at $432 \mathrm{~nm}$ every 13 ns. In Fig. 3(a), the time-resolved PL spectrum of a NS is shown as a two-dimensional (2D) image, plotted in false color on a logarithmic scale with the most intense emission indicated in red. The vertical axis of the map represents 

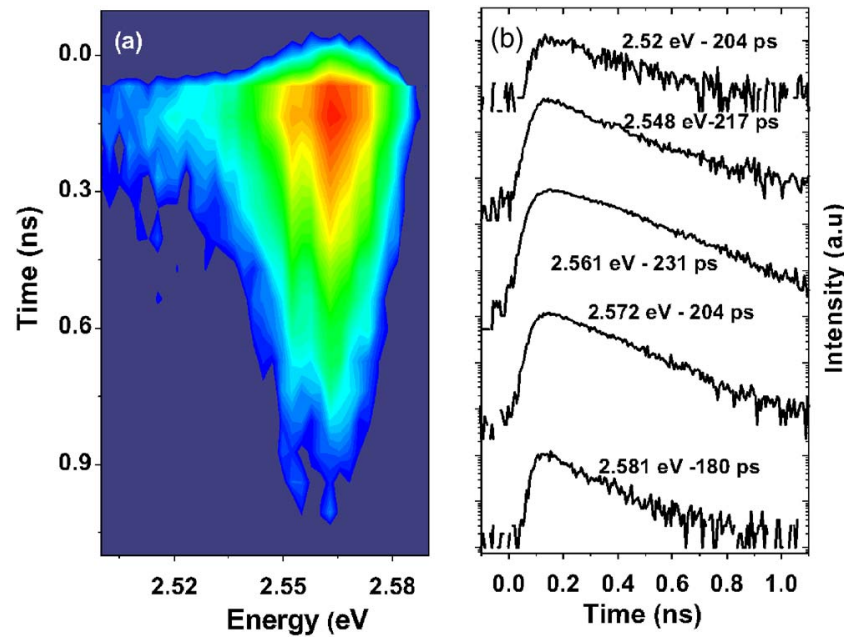

FIG. 3. (Color online) Time-resolved PL obtained from a single NS. (a) A 2D image of time vs energy and (b) time decays of PL taken at different emission energies from (a).

the time after the laser pulse, while the horizontal axis represents the emission energy. In this image, PL emission at energies 2.547 and $2.563 \mathrm{eV}\left(I_{2 A}\right.$ and $\left.I_{2 B}\right)$ are the most intense spectral features.

In Fig. 3(b) time decays are extracted from the timeresolved spectra at several different energies. The $I_{2 A}$ and $I_{2 B}$ lines exhibit time decays which are single exponential with lifetimes of approximately $200 \mathrm{ps}$. This lifetime is somewhat shorter than that measured in bulk CdS (500 ps-1 ns), ${ }^{16,17}$ but significantly longer than the near band edge emission observed in single CdS nanowires $(<50 \mathrm{ps}) .{ }^{10}$ These long lifetimes are a reflection of the quality of these CdS NSs.

In conclusion, we have shown that catalyst-assisted vapor phase growth of CdS NSs with large width-to-thickness aspect ratios results in high quality hexagonal wurtzite single crystals, as is reflected in the HR-TEM measurements. These NSs display intense near band edge emission $A$ - and $B$-related exciton emission. Time-resolved measurements reveal 200 ps lifetimes, which are much longer than CdS nanowires whose diameters are the thickness of these NSs, but somewhat shorter than those observed in bulk crystals (up to
$1 \mathrm{~ns})$. This suggests that future passivation of the NS surfaces may result in even higher quantum efficiencies. Polarization-resolved PL measurements show strongly polarized emission which reflect recombination selection rules for the wurtzite band symmetries. In particular, we find that analysis of the polarized emission axis can rapidly reveal the orientation of the $c$ axis within single NSs. We have demonstrated that the use of polarized spatially resolved spectroscopy can be important in efforts to optimize growth of these NSs to obtain highly reproducible materials which is essential for future application development.

H.R., L.V.T. and TBH are equally responsible for this work. This work was supported by the National Science Foundation by Grant Nos. EEC/NUE 0532495 and ECCS 0701703, USA, by the Korea Research Foundation Grant funded by the Korean Government (MOEHRD) (KRF-2006005-J00302), by the international collaborative research funds of Chonbuk National University, 2007, and the NSs growth by the KIST-CNRS LIA program (2U03450) and the KIST project (2E19770).

${ }^{1}$ X. F. Duan, C. M. Niu, V. Sahi, J. Chen, J. W. Parce, S. Empedocles, and J. L. Goldman, Nature (London) 425, 274 (2003).

${ }^{2}$ Z. L. Wang, Annu. Rev. Phys. Chem. 55, 159 (2004).

${ }^{3}$ L. Weifeng, J. Chong, J. Chuangui, Y. Lianzeng, C. Weili, and L. Xiaoguang, J. Cryst. Growth 269, 304 (2004).

${ }^{4}$ W. Chunrui, K. M. Ip, S. K. Hark, and L. Quan, J. Appl. Phys. 97, 054303 (2005).

${ }^{5}$ T. Gao, Q. H. Li, and T. H. Wang, Appl. Phys. Lett. 86, 173105 (2005).

${ }^{6}$ R. M. Ma, L. Dai, H. B. Huo, W. Q. Yang, G. G. Qin, P. H. Tan, C. H. Huang, and J. Zheng, Appl. Phys. Lett. 89, 203120 (2006).

${ }^{7}$ D. H. L. Ng, W. Yu, and C. Y. To, Mater. Lett. 60, 1151 (2006).

${ }^{8}$ Z. Q. Wang, J. F. Gong, J. H. Duan, H. B. Huang, S. G. Yang, X. N. Zhao,

R. Zhang, and Y. W. Du, Appl. Phys. Lett. 89, 033102 (2006).

${ }^{9}$ R. M. Ma, L. Dai, and G. G. Qin, Nano Lett. 7, 868 (2007).

${ }^{10}$ L. V. Titova, T. B. Hoang, H. E. Jackson, L. M. Smith, J. M. YarrisonRice, J. L. Lensch, and L. J. Lauhon, Appl. Phys. Lett. 89, 053119 (2006).

${ }^{11}$ T. B. Hoang, L. V. Titova, H. E. Jackson, L. M. Smith, J. M. YarrisonRice, J. L. Lensch, and L. J. Lauhon, Appl. Phys. Lett. 89, 123123 (2006).

${ }^{12}$ Y.-J. Choi, K. J. Choi, D.-W. Kim, and J.-G. Park (unpublished).

${ }^{13}$ G. D. Gilliland, Mater. Sci. Eng., R. R18, 99 (1997).

${ }^{14}$ J. J. Hopfield and D. G. Thomas, Phys. Rev. 122, 35 (1961).

${ }^{15}$ D. G. Thomas and J. J. Hopfield, Phys. Rev. 128, 2135 (1962).

${ }^{16}$ R. Heitz, A. Hoffmann, and I. Broser, Phys. Rev. B 49, 14307 (1994).

${ }^{17}$ C. H. Henry and K. Nassau, Phys. Rev. B 1, 1628 (1970). 